ARTICLE

https://doi.org/10.1038/s41467-020-15916-3

\title{
Interspecies radiative transition in warm and superdense plasma mixtures
}

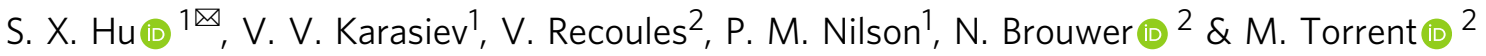

Superdense plasmas widely exist in planetary interiors and astrophysical objects such as brown-dwarf cores and white dwarfs. How atoms behave under such extreme-density conditions is not yet well understood, even in single-species plasmas. Here, we apply thermal density functional theory to investigate the radiation spectra of superdense iron-zinc plasma mixtures at mass densities of $\rho=250$ to $2000 \mathrm{~g} \mathrm{~cm}^{-3}$ and temperatures of $\mathrm{kT}=50$ to 100 $\mathrm{eV}$, accessible by double-shell-target implosions. Our ab initio calculations reveal two extreme atomic-physics phenomena-firstly, an interspecies radiative transition; and, secondly, the breaking down of the dipole-selection rule for radiative transitions in isolated atoms. Our first-principles calculations predict that for superdense plasma mixtures, both interatomic radiative transitions and dipole-forbidden transitions can become comparable to the normal intra-atomic $\mathrm{K} \alpha$-emission signal. These physics phenomena were not previously considered in detail for extreme high-density plasma mixtures at super-high energy densities.

\footnotetext{
${ }^{1}$ Laboratory for Laser Energetics, University of Rochester, 250 East River Road, Rochester, NY 14623-1299, USA. ${ }^{2}$ CEA, DAM, DIF, 91297 Arpajon, France.

凶email: shu@lle.rochester.edu
} 
E xtreme material conditions, such as super-high density and warm or hot temperatures, can be widely found in the universe. For example, brown-dwarf cores and white dwarfs ${ }^{1-3}$ can have a mass density of $\rho=10^{3}-10^{7} \mathrm{~g} \mathrm{~cm}^{-3}$ and temperatures up to $\sim 10^{6} \mathrm{~K}$. Thanks to technological advancements, such extreme states of matter can now be created in the laboratory using powerful lasers ${ }^{4-7}$ and/or pulsed-power machines $^{8}$. For instance, deuterium and tritium contained in a millimeter-size inertial confinement fusion (ICF) target can be squeezed to $\rho=10^{2}-10^{3} \mathrm{~g} \mathrm{~cm}^{-3}$ by powerful lasers through laserdriven compression and spherical convergence ${ }^{9-13}$. Using doubleshell implosions ${ }^{14,15}$, mid-/high- $Z$ materials can be squeezed to super-high densities ranging from $\rho=10^{3}-10^{4} \mathrm{~g} \mathrm{~cm}^{-3}$ with a temperature ranging from tens to hundreds of electron volts $(1 \mathrm{eV} \sim 11,604 \mathrm{~K})$. Understanding how matter behaves at such extreme conditions is the purview of high-energy-density physics, inertial confinement fusion, planetary science, and astrophysics.

Under superdense conditions, atoms and molecules-the fundamental building blocks of matter-can have drastically different properties from those found under ambient conditions. For instance, because of pressure ionization, the binding energy of core electrons of atoms might significantly shift in dense plasmas ${ }^{16-18}$ when compared with the case of isolated atoms. By probing the energy level changes in these systems, one can infer the dense-plasma conditions if one knows precisely beforehand how atoms behave in high-density environments. Moreover, such an extreme environment experienced by embedded ions can also alter the characteristics of atomic wavefunctions because of closely encountered neighboring ions. This can have profound implications for understanding radiation transport in such dense plasmas. For example, the dipole-selection rule for isolated atoms can break down in extremely dense plasmas. Most interestingly, if a plasma mixture is compressed to very high densities above $10^{3}$ $\mathrm{g} \mathrm{cm}^{-3}$, wave-function overlapping of deeply bound electrons between different atomic species may occur. A schematic diagram of such a scenario is depicted in Fig. 1, in which the iron $(\mathrm{Fe})$ and zinc $(\mathrm{Zn})$ ions in the mixture closely interact with each other in superdense plasma. As a result of the short distance $(\mathrm{d})$ between the two species, their outer electrons on $n=3$ and $n=4$ levels can be pressure ionized and their $2 s$ and $2 p$ states might also be significantly distorted by each other. The significant overlapping of $n=2$ states could enable a physics phenomenon-interspecies radiative transitions (IRT) - to occur.

As Fig. 1 illustrates, if $1 s$ holes of both $\mathrm{Fe}$ and $\mathrm{Zn}$ ions are created by either radiation pumping ${ }^{19}$ or energetic electron collisions $^{20,21}$, the $2 s$ and $2 p$ electrons of one species (e.g., $\mathrm{Fe}$ ) could radiatively transition to the $1 s$ hole of the other species (e.g., $\mathrm{Zn}$ ), giving interspecies $\mathrm{K} \alpha$ emission. On the other hand, if the $2 p$ state is no longer fully occupied and the $1 s$-core state is filled, the interspecies $\mathrm{K} \alpha$ absorption could occur in such extremely denseplasma mixtures. To the best of our knowledge, this phenomenon of IRT between bound states has not been considered in emissivity/opacity calculations of plasma mixtures ${ }^{22-26}$, even though inter-Coulombic Auger decay was discovered in large molecules and clusters ${ }^{27-29}$, and collision-induced absorption and emission between atomic gases was discussed ${ }^{30-32}$. Furthermore, the significant distortion of the $2 s$ state resulting from closest neighboring ions will make both intra-atomic and inter-atomic $2 s-1 s$ transitions possible, which are dipole-forbidden for an isolated atom and relatively low-density systems when deeply bound $2 s$ and $1 s$ states preserve their ideal $s$ symmetry. To classify various transitions, we use the word of intra-atomic for transitions of electron having both initial state and final state belong to the same atom, while inter-atomic transitions involve two atoms that can be either the same type or different species.

Here, we present interspecies radiative transition results from first-principles calculations by thermal density-functional theory (DFT) using the ABINIT software package 33,34 in the planewave-based projector augmented-wave (PAW) approach. All electrons are considered as evolving-no frozen core approximation-and spin-orbit coupling effects are explicitly included. As an example for mid- $Z$ elements presented in brown-dwarf cores, a dense-plasma mixture of $\mathrm{Fe}$ and $\mathrm{Zn}$ was considered with an equal atomic fraction for each species (50:50). We varied the $\mathrm{Fe}-\mathrm{Zn}$ plasma density from $\rho=250$ to $2000 \mathrm{~g} \mathrm{~cm}^{-3}$ and temperatures of $\mathrm{kT}=50$ to $100 \mathrm{eV}$. For a chosen plasma condition, we first ran orbital-free DFT-based molecular dynamics ${ }^{35,36}$ to obtain the equilibrium ionic configurations. We then took several snapshots of uncorrelated ionic configuration for the electronic structure calculations using ABINIT. Once the electronic structure of a dense plasma is determined from the ABINIT calculations, we created a $1 s$-hole state by removing the occupation of the $1 s$ state for both $\mathrm{Fe}$ and $\mathrm{Zn}$ ions. Finally, we calculated the dipole matrices to determine the emission spectra of superdense $\mathrm{Fe}-\mathrm{Zn}$ plasmas with the Kubo-Greenwood formalism. More numerical details and convergence tests can be found in the "Methods" and Supplementary Information.

\section{Results}

Interspecies radiative transition in warm and superdense plasmas. For a superdense and warm Fe-Zn plasma of $\rho=1000$ $\mathrm{g} \mathrm{cm}^{-3}$ and $\mathrm{kT}=50 \mathrm{eV}$ with $1 s$ vacancies of both $\mathrm{Fe}$ and $\mathrm{Zn}$ ions, the calculated emission coefficient as a function of photon energy

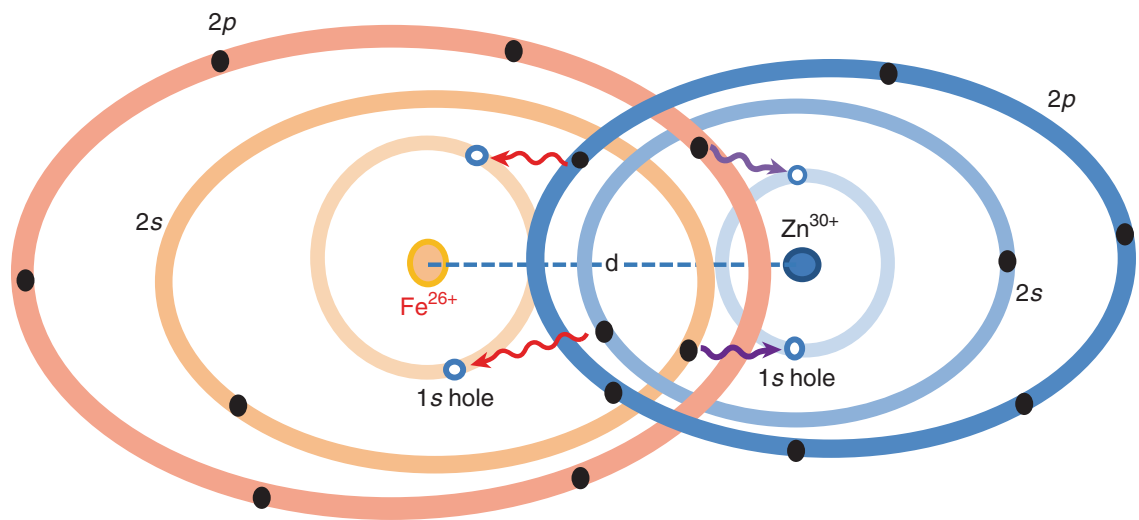

Fig. 1 The schematic diagram of interspecies radiative transition in superdense plasmas. Due to the high compression of superdense plasmas, ions of different species can get so close to each other that their atomic states may become overlapping. In such a superdense environment, photon-pumping or collision-induced $1 s$-core holes can be filled by $2 p$ electrons from other species, giving rise to inter-atomic K $\alpha$ emission. 


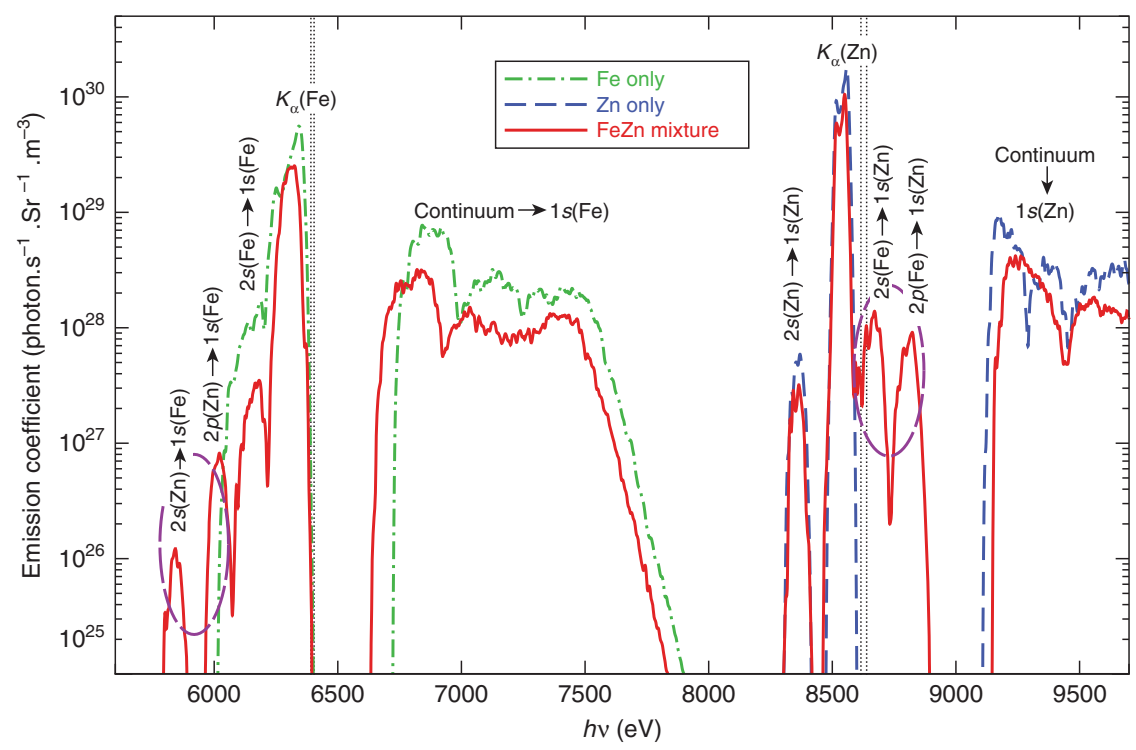

Fig. 2 The emission spectra of superdense plasmas. Three cases are calculated for Fe-only, Zn-only, and Fe-Zn mixture having $1 \mathrm{~s}$ vacancy at $\rho=1000 \mathrm{~g}$ $\mathrm{cm}^{-3}$ and $\mathrm{kT}=50 \mathrm{eV}$, using density-functional theory (DFT) with ABINIT.

is shown by the solid-red line in Fig. 2. To identify the IRT features, we also plotted the spectra of single-species $\mathrm{Fe}$ (dashed-dotted green line) and $\mathrm{Zn}$ (dashed blue line) plasmas in Fig. 2, respectively. Again, these pure plasmas have the same density and temperature conditions as that of the Fe-Zn mixture.

From Fig. 2, one can clearly see that four additional spectral peaks appear in the superdense $\mathrm{Fe}-\mathrm{Zn}$ plasma mixtures (highlighted by the dashed ellipse): the two emission lines located at hv $\approx 8666 \mathrm{eV}$ and $\mathrm{h} v \approx 8816 \mathrm{eV}$ correspond to transitions from the $2 s$ and $2 p$ states of the $\mathrm{Fe}$ ion to the $1 s$ hole of the $\mathrm{Zn}$ ion, while the other two peaks at $\mathrm{h} v \approx 5838 \mathrm{eV}$ and $\mathrm{h} v \approx 6012 \mathrm{eV}$ belong to radiative transitions of $2 s / 2 p$ electrons of the $\mathrm{Zn}$ ion to the $1 s$ vacancy of $\mathrm{Fe}$. Besides these inter-atomic $\mathrm{K} \alpha$ emissions, the dominant intra-atomic $\mathrm{K} \alpha$ lines for each species, are of course, present in the emission spectra in Fig. 2. The vertical dotted black lines mark the normal intra-atomic $\mathrm{K} \alpha$ locations of ambient $\mathrm{Fe}$ and $\mathrm{Zn}$, respectively. The red shift of the intra-atomic $\mathrm{K} \alpha$ line is caused by the increased electron screening resulting from the dense plasma environment ${ }^{19}$. In addition, the intra-atomic $2 s \rightarrow$ $1 s$ transitions for each species, although being about three orders of magnitude weaker than the normal intra-atomic K $\alpha$ lines, also appear as a consequence of the breaking down of the dipoleselection rule due to the density-induced distortion of $2 s$ states. Finally, the continuum emissions from free electrons filling $1 \mathrm{~s}$ holes of $\mathrm{Fe}$ and $\mathrm{Zn}$ ions are also present in the emission spectra, as expected (shown by Fig. 2).

To further understand the emission spectra of Fig. 2, we have computed the density of states (DOS) for the three denseplasma cases. The results are plotted in Fig. 3, in which Fig. 3a, $3 \mathrm{~b}$ are for Fe-only and $\mathrm{Zn}$-only plasmas, respectively. One finds that the outer bound states of $3 s, 3 p, 3 d$ (or $4 s$ ) states of $\mathrm{Fe}$ and $\mathrm{Zn}$ atoms have merged into the continuum because of pressure and thermal ionization. Note that the continuum states below and above the Fermi level $\left(\mathrm{E}_{\mathrm{F}}\right)$ (i.e., chemical potential) are partially occupied. Clearly, the discrete states of $1 s, 2 s$, and $2 p$ of $\mathrm{Fe}$ and $\mathrm{Zn}$ ions are evidenced in Fig. 3a, 3b. By looking into the occupations on states below the Fermi energy, the estimated average ionizations are $\langle Z\rangle \approx 17.3$ and $\langle Z\rangle \approx 19.1$, respectively, for Fe-only and $\mathrm{Zn}$-only cases. This indicates that the $2 p$ state of Fe begins to be partially occupied. When Fe and $\mathrm{Zn}$ plasmas are mixed together, their discrete states of $1 s, 2 s$, and $2 p$ are slightly $\mathrm{red} / \mathrm{blue}$ shifted by $\sim 15$ to $30 \mathrm{eV}$ in Fig. $3 \mathrm{c}$ when compared with the corresponding pure-plasma cases. This shift can be attributed to the interactions between the two species. Now, if their $1 s$ states become empty, i.e. a hole/vacancy is created, the radiative transitions from $2 s / 2 p$ electrons of $\mathrm{Fe}$ and $\mathrm{Zn}$ ions to fill $1 s$ holes give rise to the corresponding emission lines in Fig. 2. The breaking down of dipole-selection rule for the $2 s \rightarrow$ $1 s$ transitions is caused by non-spherical character in the $2 s$ state due to density-induced distortions. Finally, the emission from transitions of continuum to the $1 \mathrm{~s}$ hole can also be explained.

Density dependence of interspecies radiative transition (IRT). To explore how density change affects the interspecies radiative transition in superdense plasmas, we have performed similar first-principles calculations by varying the $\mathrm{Fe}-\mathrm{Zn}$ density from $\rho=250 \mathrm{~g} \mathrm{~cm}^{-3}$ to $\rho=2000 \mathrm{~g} \mathrm{~cm}^{-3}$ but keeping $\mathrm{kT}=50 \mathrm{eV}$. The DFT-predicted emission spectra are plotted in Fig. 4 for three different $\mathrm{Fe}-\mathrm{Zn}$ plasma densities of $\rho=500,1000$, and $1500 \mathrm{~g} \mathrm{~cm}^{-3}$. Again, the IRT peaks are highlighted by the dashed ellipses in each panel of the figure.

At a relatively lower density of $\rho=500 \mathrm{~g} \mathrm{~cm}^{-3}$, Fig. 4a shows that the inter-atomic $\mathrm{K} \alpha$ emission is significantly weaker than the normal intra-atomic $\mathrm{K} \alpha$ emission by $\sim 3$ to 4 orders of magnitude. They are even lower than that of dipole-forbidden intra-atomic $2 s \rightarrow 1 s$ transitions. It is noted that the spin-orbit coupling-induced splitting of $K \alpha 1$ and $K \alpha 2$ is clearly seen for $\mathrm{Zn}$, but for Fe they are merged into one peak because of density/ temperature broadenings. As the $\mathrm{Fe}-\mathrm{Zn}$ plasma density increases to $\rho=1000 \mathrm{~g} \mathrm{~cm}^{-3}$ and $\rho=1500 \mathrm{~g} \mathrm{~cm}^{-3}$, the inter-atomic $\mathrm{K} \alpha$ emission peaks drastically rise in amplitude and their widths increase as a result of strong density broadening (Fig. 4b, c). At an extremely high $\mathrm{Fe}-\mathrm{Zn}$ density of $\rho=1500 \mathrm{~g} \mathrm{~cm}^{-3}$, Fig. $4 \mathrm{c}$ indicates that the peak amplitude of inter-atomic $\mathrm{K} \alpha$ from $2 p$ $(\mathrm{Fe}) \rightarrow 1 s(\mathrm{Zn})$ transition can approach $\sim 10 \%$ of the intra-atomic $\mathrm{K} \alpha$ from $2 p(\mathrm{Zn}) \rightarrow 1 s(\mathrm{Zn})$ transition, which should be readily detectable in experiments. It is noted that these inter-atomic transitions become even stronger than the dipole-forbidden intraatomic $2 s(\mathrm{Zn}) \rightarrow 1 s(\mathrm{Zn})$ transition.

One interesting feature seen in Fig. 4 is that the inter-atomic $\mathrm{K} \alpha$ emission from $2 p(\mathrm{Fe}) \rightarrow 1 s(\mathrm{Zn})$ transition is always stronger than that of $2 p(\mathrm{Zn}) \rightarrow 1 s(\mathrm{Fe})$. To further explore this asymmetry and the overall trend of IRT versus plasma density, we have 

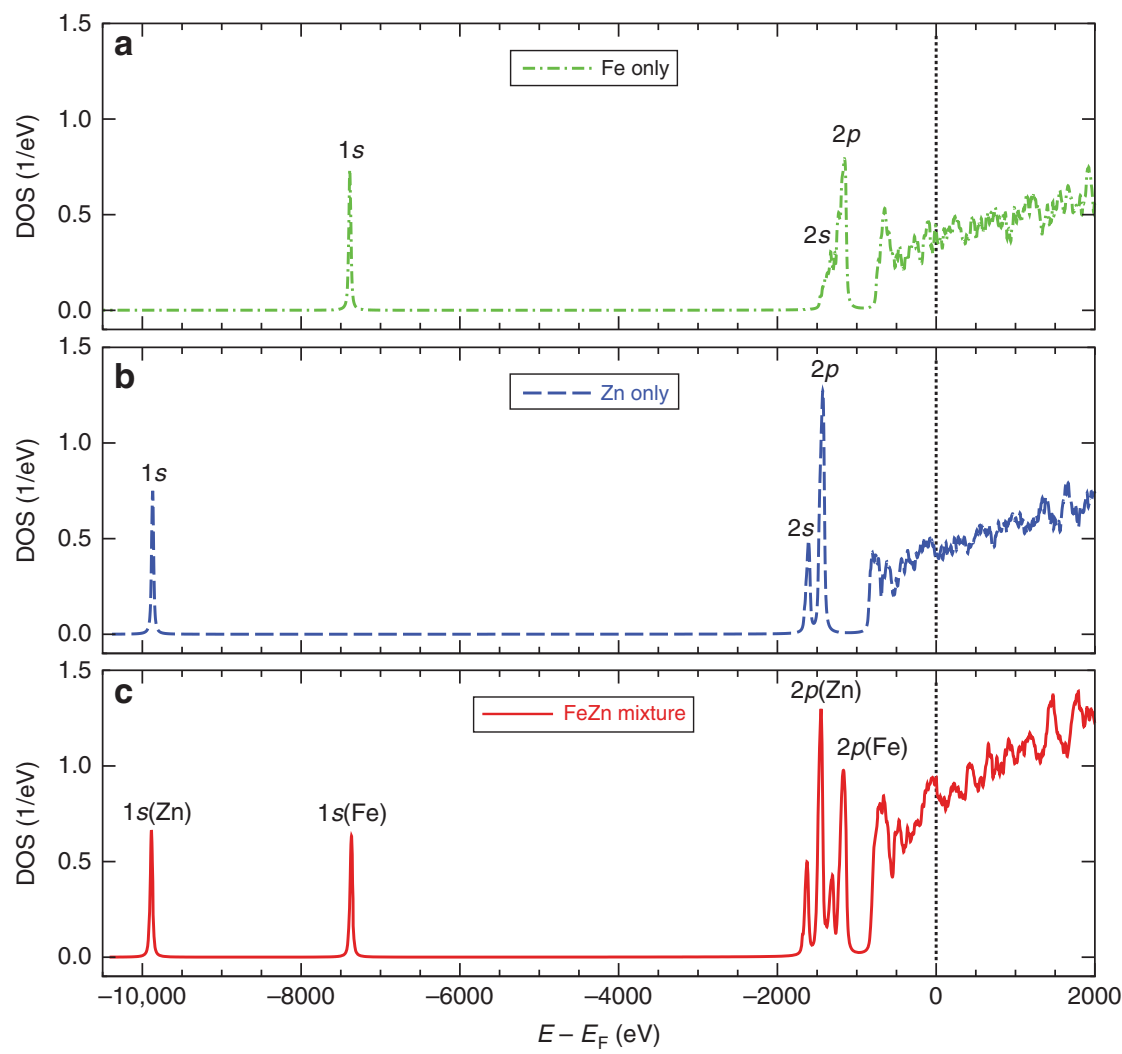

Fig. 3 The density of states (DOS) of superdense plasmas. a Pure Fe plasmas, $\mathbf{b}$ pure $\mathrm{Zn}$ plasmas, and $\mathbf{c}$ Fe-Zn mixture plasmas at the same density and temperature of $\rho=1000 \mathrm{~g} \mathrm{~cm}^{-3}$ and $\mathrm{kT}=50 \mathrm{eV}$.
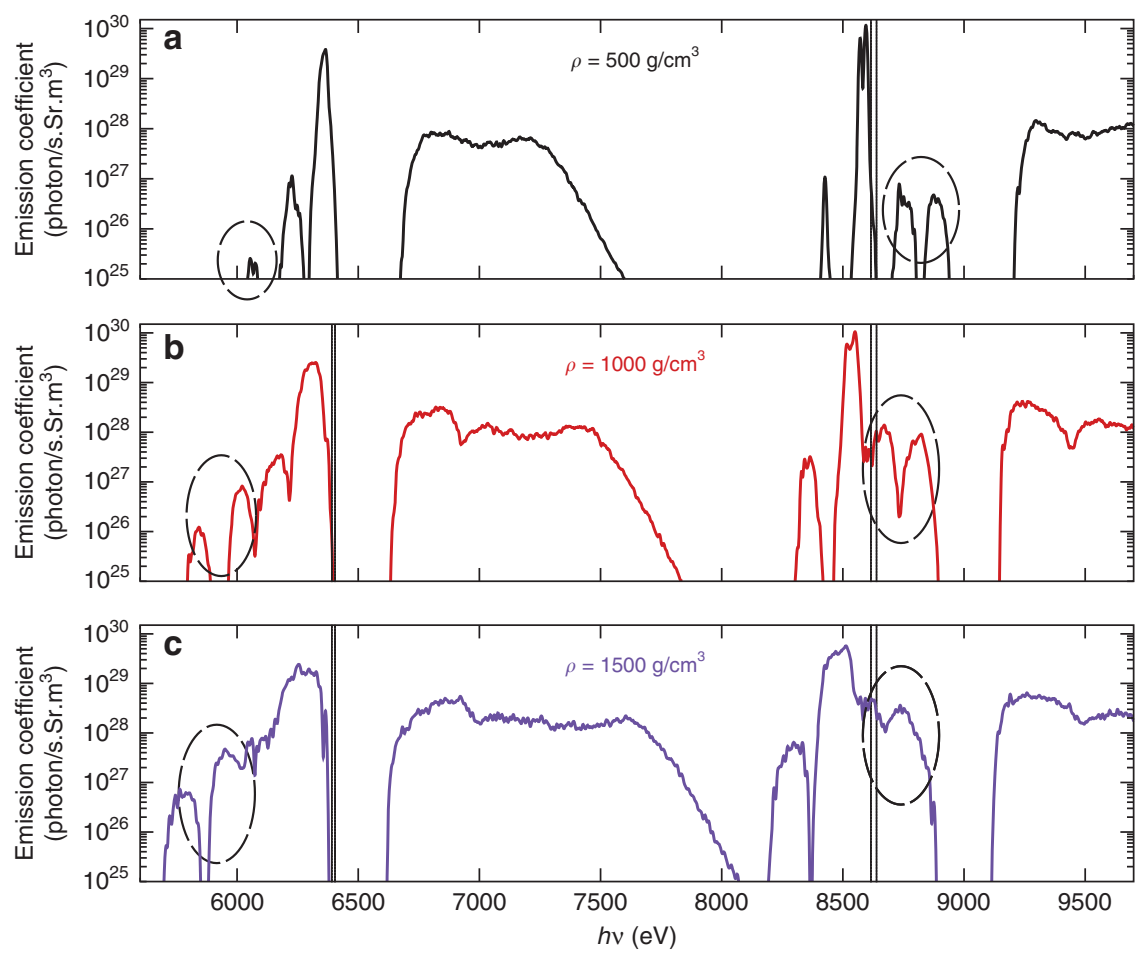

Fig. 4 Interspecies radiative transitions (IRT) versus plasma density. DFT calculations are done for superdense Fe-Zn mixtures at $\mathrm{kT}=50 \mathrm{eV}$ but different plasma mass densities: $\mathbf{a} \rho=500 \mathrm{~g} \mathrm{~cm}^{-3}, \mathbf{b} \rho=1000 \mathrm{~g} \mathrm{~cm}^{-3}$, and $\mathbf{c} \rho=1500 \mathrm{~g} \mathrm{~cm}^{-3}$. 

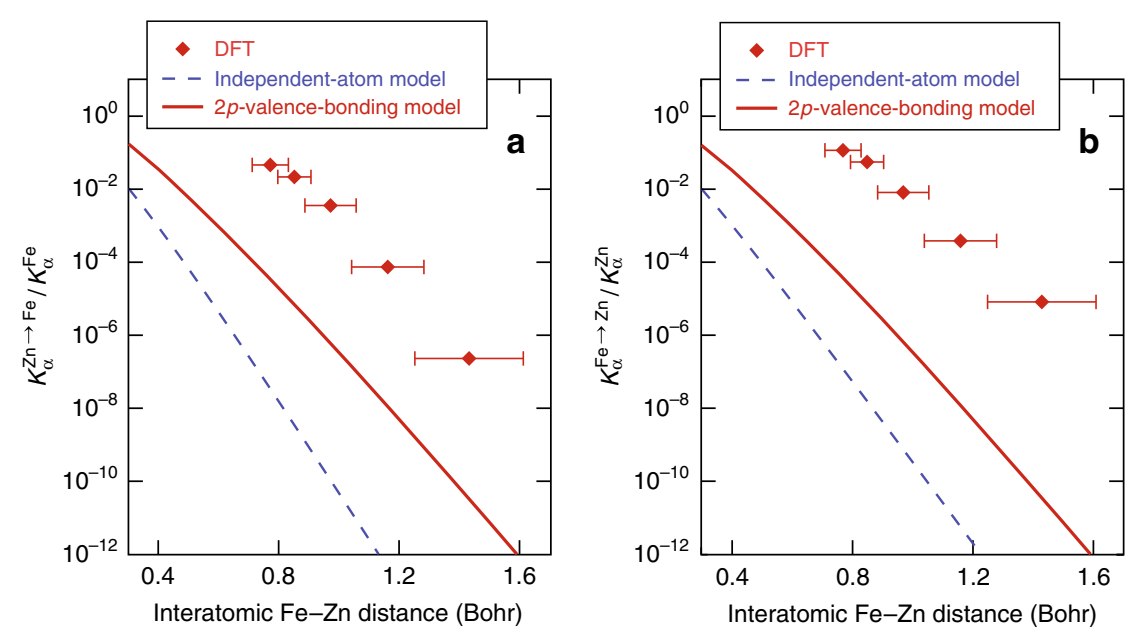

Fig. 5 Model predictions of IRT in comparison with DFT calculations. a The ratio of inter-atomic K $\alpha$ emission (from $\mathrm{Zn}$ to $\mathrm{Fe}$ ) to the regular intra-atomic $\mathrm{K} \alpha$ signal (within Fe) as a function of inter-atomic Fe-Zn distance. b Similar to $\mathbf{a}$ but for inter-atomic K $\alpha$ emission from Fe to $\mathrm{Zn}$. All of these calculations were done at different plasma densities with the same temperature of $\mathrm{kT}=50 \mathrm{eV}$. DFT stands for density-functional theory. The "error bar" in DFT data represents the full-width half-maximum of $g(r)$ peak.

plotted the DFT-predicted ratio of the inter-atomic $\mathrm{K} \alpha$ signal $\mathrm{K}_{\alpha}^{\mathrm{Zn} \rightarrow \mathrm{Fe}}$ or $\mathrm{K}_{\alpha}^{\mathrm{Fe} \rightarrow \mathrm{Zn}}$ to the corresponding intra-atomic emission $\mathrm{K}_{\alpha}^{\mathrm{Fe}}\left(\right.$ or $\left.\mathrm{K}_{\alpha}^{\mathrm{Zn}}\right)$ (by the red diamond symbols in Fig. 5) as a function of inter-atomic distance between $\mathrm{Fe}$ and $\mathrm{Zn}$ ions. For a chosen Fe-Zn plasma density varying from $\rho=250 \mathrm{~g} \mathrm{~cm}^{-3}$ to $\rho=2000$ $\mathrm{g} \mathrm{cm}^{-3}$ at the same temperature of $\mathrm{kT}=50 \mathrm{eV}$, we derived the Fe-Zn distance (d) from the orbital-free DFT-MD runs, in which $d$ corresponds to the peak location of the pair distribution function $-g(r)$. The full-width half-maximum of $g(r)$ peak gives the plausible range of Fe- $\mathrm{Zn}$ distance ("error bar" of $\mathrm{d}$ in Fig. 5).

To further see how the inter-atomic K $\alpha$ emission qualitatively changes with $d$, we have used two simple models to estimate the inter-to-intra $\mathrm{K} \alpha$ ratio through the following dipole-matrix elements calculations:

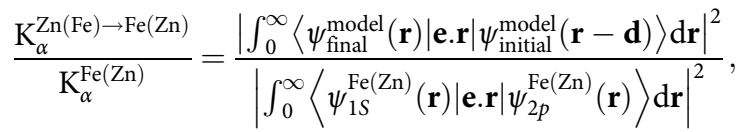

where the unit vector e defines the direction of dipole moment. Depending on how we choose the initial and final states in the nominator of the above equation to estimate the matrix element, we have two simple models: (a) the independent-atom model (IAM) which simply takes the $2 p$ and $1 s$ hydrogenic wavefunctions of independent $\mathrm{Fe}$ and $\mathrm{Zn}$ atoms as the initial and final states (namely $\left|\psi_{\text {initial }}^{\mathrm{IAM}}(\mathbf{r})\right\rangle=\left|\psi_{2 p}^{\mathrm{Zn}(\mathrm{Fe})}(\mathbf{r})\right\rangle$ and $\left|\psi_{\text {final }}^{\mathrm{IAM}}(\mathbf{r})\right\rangle=$ $\left|\psi_{1 s}^{\mathrm{Fe}(\mathrm{Zn})}(\mathbf{r})\right\rangle$.); and (b) the 2p-valence-bonding model in which the initial and final states have a form of symmetrized products of hydrogenic $1 s$ and $2 p$ wavefunctions of both $\mathrm{Fe}$ and $\mathrm{Zn}$ atoms, that is, $\left|\psi_{\text {initial }}^{2 \text { p-valence-bonding }}\left(\mathbf{r}_{1}, \mathbf{r}_{2}\right)\right\rangle=\left[\left|\psi_{2 p}^{\mathrm{Fe}}\left(\mathbf{r}_{1}\right)\right\rangle\left|\psi_{2 p}^{\mathrm{Zn}}\left(\mathbf{r}_{2}\right)\right\rangle+\right.$ $\left.\left|\psi_{2 p}^{\mathrm{Fe}}\left(\mathbf{r}_{2}\right)\right\rangle\left|\psi_{2 p}^{\mathrm{Zn}}\left(\mathbf{r}_{1}\right)\right\rangle\right] / \sqrt{2} \quad$ and $\quad\left|\psi_{\text {final }}^{2 \mathrm{p}-\text { valence-bonding }}\left(\mathbf{r}_{1}, \mathbf{r}_{2}\right)\right\rangle=$ $\left[\left|\psi_{1 s}^{\mathrm{Fe}(\mathrm{Zn})}\left(\mathbf{r}_{1}\right)\right\rangle\left|\psi_{2 p}^{\mathrm{Zn}(\mathrm{Fe})}\left(\mathbf{r}_{2}\right)\right\rangle+\left|\psi_{1 s}^{\mathrm{Fe}(\mathrm{Zn})}\left(\mathbf{r}_{2}\right)\right\rangle\left|\psi_{2 p}^{\mathrm{Zn}(\mathrm{Fe})}\left(\mathbf{r}_{1}\right)\right\rangle\right] / \sqrt{2}$, which is analog to the covalent bonding in molecules. For the latter model, six-dimensional integration over $\mathbf{r}_{1}$ and $\mathbf{r}_{2}$ is needed for evaluating the dipole matrix. Using these two models, we can qualitatively estimate the above ratios as a function of the inter-atomic distance $d$. The results are plotted as the dashed-blue and solid-red lines in Fig. 5a and b, respectively, for the independent-atom model and the $2 \mathrm{p}$ valence-bonding model. One can see that these simple models qualitatively give the overall trend of increasing inter-atomic $\mathrm{K} \alpha$ emission as inter-atomic distance decreasing; Quantitatively, both models show orders of magnitude differences from manybody DFT calculations. Nevertheless, the $2 \mathrm{p}$-valence-bonding model is better than the independent-atom model, which manifests the molecular-bonding nature among atoms in such superdense systems. Molecular bonding involving more than two atoms might account for the discrepancies between the two simple models and DFT calculations. It is noted that the multicenter wave-function nature ${ }^{37}$ was properly accounted for by DFT. At the highest density explored $\left(\rho=2000 \mathrm{~g} \mathrm{~cm}^{-3}\right)$, Fig. $5 \mathrm{~b}$ shows that the inter-atomic $\mathrm{K}_{\alpha}^{\mathrm{Fe} \rightarrow \mathrm{Zn}}$ emission can reach over $11 \%$ of the regular intra-atomic $\mathrm{K}_{\alpha}^{\mathrm{Zn}}$ signal. The asymmetry that the inter-atomic $\mathrm{K} \alpha$ emission from $2 p(\mathrm{Zn}) \rightarrow 1 s(\mathrm{Fe})$ transition is always weaker than that of $2 p(\mathrm{Fe}) \rightarrow 1 s(\mathrm{Zn})$ is caused by the fact that the $2 p$ state of Fe ion $\left[\mid \psi_{2 p}^{\mathrm{Fe}}(\mathbf{r})>\right]$ spreads much more than $\mid \psi_{2 p}^{\mathrm{Zn}}(\mathbf{r})>$ (because the former is less bounded) so that it can have significant overlap with the $1 s$ hole of $\mathrm{Zn}$ (see Fig. 1). This consideration is further confirmed by looking into the orbital (wave-function) overlap in such superdense situations.

Finally, we shall discuss the radiative-to-nonradiative decay branching ratio for 1s-core-hole states created in such superdense-plasma mixtures. We shall point out that for superdense plasmas considered here, the nonradiative Auger decay channel is hard to measure because Auger electrons will quickly thermalize inside the superdense plasma; On the other hand, the radiative decay can be easily probed by measuring the escaped $\mathrm{K}_{\alpha}$ photons through spectrometers. To calculate the ratio of $\mathrm{K}_{\alpha}$ emission to Auger decay, we have used the atomic kinetic modeling code PrimSPECT ${ }^{38}$, which is extensively used in the plasma physics community. For the concerned plasma densities varying from $250 \mathrm{~g} \mathrm{~cm}^{-3}$ to $2000 \mathrm{~g} \mathrm{~cm}^{-3}$ and $\mathrm{kT}=50 \mathrm{eV}$, the averaged ionizations of $\mathrm{Fe}$ and $\mathrm{Zn}$ ions are about $\langle\mathrm{Z}\rangle=$ 15.8 16.2 and $\langle Z\rangle=19.5 \sim 20.1$, respectively. Namely, such superdense plasmas mainly consist of neon-like ions of $\mathrm{Fe}^{16+}$ and $\mathrm{Zn}^{20+}$, which both have the dominant electronic configuration of $1 s^{2} 2 s^{2} 2 p^{6}$ that is close to what is shown by Fig. 3. Now, if external radiative/collisional pump creates 1s-core-hole state 
$\left(1 s^{1} 2 s^{2} 2 p^{6}\right)$ of both ions, we can use PrimSPECT to compute the decay rate coefficients. The calculations give a decay rate of $\Gamma_{\text {rad }}=$ $5.4 \times 10^{14} \mathrm{~s}^{-1}$ for the radiative channel of $1 s^{1} 2 \mathrm{~s}^{2} 2 \mathrm{p}^{6} \rightarrow 1 \mathrm{~s}^{2} 2 \mathrm{~s}^{2} 2 \mathrm{p}^{5}$ $\left(\mathrm{K}_{\alpha}\right.$ emission) for $\mathrm{Fe}^{17+}$ ions, while its Auger decay rate is about $\Gamma_{\text {Auger }}=9.8 \times 10^{14} \mathrm{~s}^{-1}$ for the dominant transition of $1 \mathrm{~s}^{1} 2 \mathrm{~s}^{2} 2 \mathrm{p}^{6}$ $\rightarrow 1 s^{2} 2 s^{2} 2 p^{4}$. Thus, the radiative-to-Auger branching ratio for $\mathrm{Fe}^{17+}$ ions is about $\Gamma_{\text {rad }} / \Gamma_{\text {Auger }} \approx 0.55$. For $\mathrm{Zn}^{21+}$ ions, PrimSPECT calculations give the two decay rate coefficients of $\Gamma_{\text {rad }}=1.01 \times 10^{15} \mathrm{~s}^{-1}$ and $\Gamma_{\text {Auger }}=1.07 \times 10^{15} \mathrm{~s}^{-1}$, respectively, which results in a branching ratio of $\Gamma_{\text {rad }} / \Gamma_{\text {Auger }} \approx 0.94$. These calculations indicate that the radiative decay channel has the same order of probability as the nonradiative Auger decay for intra-atomic transitions. In other words, one third of $\mathrm{Fe}^{17+}$ corehole ions will decay radiatively, while one half of $\mathrm{Zn}^{21+}$ core-hole ions will emit $\mathrm{K}_{\alpha}$ photons through intra-atomic transitions. Given the same physics nature of radiative versus nonradiative decay for both intra-atomic and inter-atomic transitions, we expect the similar branching ratio should hold between the inter-atomic radiative transition and the inter-atomic Coulombic decay ${ }^{39-41}$. Once again, the inter-atomic Coulombic decays ${ }^{39-41}$ certainly occur within such superdense plasmas, although they may not be measured as easily as the inter-atomic radiative transitions.

Possible experiments on inter-atomic $\mathrm{K} \alpha$ emissions. Experimental verification of these first-principles predictions of interatomic $\mathrm{K} \alpha$ emissions can possibly be conducted at the Omega Laser Facility utilizing the platform of double-shell implosions ${ }^{14,15,42,43}$. In a double-shell target, the inner metal shell can be made of mid- $Z \mathrm{Fe}-\mathrm{Cu}$ or $\mathrm{Fe}-\mathrm{Zn}$ alloys with a core of $\mathrm{D} 2$-gas fill. When a low- $Z$ outer shell (beryllium or polystyrene) is driven symmetrically by the 60-beam OMEGA laser to spherically impact on the inner shell, it can cause the inner Fe-Zn (or $\mathrm{Fe}-\mathrm{Cu}$ ) shell to implode. A small convergence ratio of $\mathrm{C}_{\mathrm{R}}=$ $R_{\text {initial }} / R_{\text {final }} \sim 8-10$ of the metal shell could give rise to a mass density of $\rho=500$ to $2000 \mathrm{~g} \mathrm{~cm}^{-3}$ for the inner $\mathrm{Fe}-\mathrm{Zn}$ (or Fe-Cu) shell at its stagnation ${ }^{42,43}$. To create the $1 s$ holes of $\mathrm{Fe}$ and $\mathrm{Zn} / \mathrm{Cu}$ ions, one option is to use the high-intensity OMEGA EP beam to generate $\mathrm{MeV}$ electrons that can remove some of the $1 s$ electrons of $\mathrm{Fe}$ and $\mathrm{Zn} / \mathrm{Cu}$ ions through collisions. The other option is to fill the double-shell target with mid- $Z$ gases, such as Ar and $\mathrm{Kr}$. As a result, the hot-spot self-emission with a certain amount of hard $\mathrm{x}$-rays could ionize the $1 s$ electrons of $\mathrm{Fe}$ and $\mathrm{Zn} / \mathrm{Cu}$ ions by radiation pumping. The latter method has been successfully demonstrated in single-shell implosions on OMEGA. In both ways, the created hollow $\mathrm{Fe} / \mathrm{Zn} / \mathrm{Cu}$ ions in such extremely dense plasmas will give rise to inter-atomic $\mathrm{K} \alpha$ emissions, as we have predicted here. These inter-atomic K $\alpha$ emissions can be measured by spectrometers with a dynamic range of 100 to 1000 .

\section{Discussion}

The two phenomena predicted from our first-principles DFT calculations, which are the interspecies radiative transition and the breaking down of dipole-selection rule in extremely denseplasma mixtures, can have significant implications to highenergy-density (HED) science, ICF, and astrophysics. For plasma opacity/emissivity calculations, the cross-talk between different species and dipole-forbidden transitions have generally been ignored so far by the HED science community. Our firstprinciples results show that these inter-atomic radiative transitions can become significant and even comparable with normal intra-atomic transitions. The overall trend of IRT can be qualitatively understood by the independent atom model and the $2 \mathrm{p}$ valence-bonding model; while transient multi-atom molecular bonding could account for the enhancement of IRT in superdense plasmas. One would expect that these emission/absorption channels, opened up in the warm and extremely dense regime, could affect the radiation transport in ICF (e.g., double-shell targets) and astrophysical objects such as brown-dwarf cores. It is noted that the inter-atomic radiative transitions shall occur in superdense single-species plasmas, although they might be indistinguishable to the normal intra-atomic transitions.

\section{Methods}

Our DFT calculations were performed with the ABINIT software package 33,34 , in which electrons are treated quantum-mechanically with a plane-wave finite-temperature Kohn-Sham DFT description. The electrons and ions are in thermodynamic equilibrium with an equal temperature $(\mathrm{Te}=\mathrm{Ti})$. The electron-nucleus interaction is described in the PAW approach by a pseudopotential generated with a very small matching radius $(\mathrm{rc}=0.2 \mathrm{bohr}$ ). All electronic wavefunctions are explicitly computed in the thermal DFT formalism. For the electronic exchange and correlation interactions, we use the generalized gradient approximation (GGA) with the

Perdew-Burke-Ernzerhof (PBE) functional ${ }^{44}$. It is noted that the PBE functional has been widely used in DFT calculations for warm-/hot-dense plasmas ${ }^{12,45-47}$ that showed good agreements with HED experiments; Our results presented here are insensitive to the choice of exchange-correlation functional, for which the localdensity approximation (LDA) gives essentially identical results, except for small energy shifts (see Supplementary Information). To sample the dense-plasma configurations, we have conducted molecular-dynamics simulations based on orbital-free DFT. Namely, under the Born-Oppenheimer approximation, the self-consistent electron density is first determined for an ion configuration. Then, the classical ions are moved by the combined electronic and ionic forces, using Newton's equation. This molecular-dynamics procedure is repeated for thousands of time steps, from which optical property (X-ray emission/absorption) can be directly evaluated. Note that we have applied the periodic boundary condition to our first-principles calculations, with a box size determined by the $\mathrm{Fe}-\mathrm{Zn}$ density and the number of atoms used. Convergent results for $\mathrm{K} \alpha$ emissions were reached by using 32 atoms in a super cell, the Baldereschi mean value point for the Brillouin zone sampling ${ }^{48}$, and the highest plane-wave energy cutoff of Ecut $\approx 68 \mathrm{keV}$. This high-energy cutoff is necessary to accurately sample the deeply bound 1s-core electrons. Detailed convergence tests can be found in the Supplementary Information.

After we ran the calculations for thousands of OFMD steps, we obtained a sufficiently long trajectory of ionic configurations. We then chose several uncorrelated snapshots from these ionic configurations to calculate the X-ray emission spectra of dense $\mathrm{Fe}-\mathrm{Zn}$ plasmas by using the Kubo-Greenwood formalism ${ }^{49,50}$. Because of the underestimated bandgap by the PBE functional due to electron selfinteraction, the resulting spectra were shifted by a constant of $\delta \omega \approx 110 \mathrm{eV}(\sim 1.5 \%$ of the $1 s-2 p$ bandgap) to match the K $\alpha$ locations of ambient $\mathrm{Fe}$ and $\mathrm{Zn}$. This is justified by comparing the Hartree-Fock calculated energy $1 s-2 p$ gap with the PBEDFT results. The similar matching technique has shown to work well for the measured $\mathrm{K} \alpha$ emission in warm dense $\mathrm{Cu}$ experiments on OMEGA EP.

In the $\mathrm{K}_{\alpha}$-emission calculations, the dipole approximation has been invoked. For the concerned photon energy range of $h v=6.0-8.8 \mathrm{keV}$, the corresponding electromagnetic waves have wavelengths of $\lambda \approx 1.4-2.1 \AA$ (2.6-3.97 Bohr). Taking an isolated $\mathrm{Fe}$ atom as an example, Hartree-Fock calculations give a size of $2 s$ and $2 p$ states $(<2 s|\mathrm{r}| 2 s>$ or $<2 \mathrm{p}|\mathrm{r}| 2 \mathrm{p}>)$ about $\sim 0.12-0.14 \AA$, which is one order of magnitude smaller than the wavelength of $\mathrm{K} \alpha$ emissions so that the dipole approximation holds well for intra-atomic transitions. For inter-atomic $\mathrm{K}_{\alpha}$ emissions in superdense $\mathrm{Fe}-\mathrm{Zn}$ plasmas $\left(\rho \geq 1000 \mathrm{~g} \mathrm{~cm}^{-3}\right)$ concerned here, the interatomic $\mathrm{Fe}-\mathrm{Zn}$ distance is around $\mathrm{d}=0.8-1.0 \mathrm{Bohr}$. Taking this emitting entity of $\mathrm{Fe}-\mathrm{Zn}$ as a whole, its size is still about $\sim 3-5$ times smaller than the $\mathrm{K} \alpha$ wavelength. Nevertheless, this prompts us to consider high-order contributions such as the electric quadrupole emission, which is examined by computing the contribution of electric quadrupole term with the independent-atom model for different densities (i.e., different inter-atomic Fe- $\mathrm{Zn}$ distances). The results indicated that the relative contribution ratio of quadrupole to dipole is overall less than $\sim 3.2 \%$ (see Supplementary Information).

\section{Data availability}

The data that support the findings of this study are available from the corresponding author upon request. They can be immediately shared through email or any other filesharing systems.

\section{Code availability}

The codes for $\mathrm{K}_{\alpha}$-emission calculations and IRT models are available from the corresponding author upon request.

Received: 25 November 2019; Accepted: 3 April 2020; Published online: 24 April 2020 


\section{References}

1. Burrows, A., Hubbard, W. B., Lunine, J. I. \& Liebert, J. The theory of brown dwarfs and extrasolar giant planets. Rev. Mod. Phys. 73, 719-765 (2001).

2. Fontaine, G., Brassard, P. \& Bergerson, P. The potential of white dwarf cosmochronology. Publ. Astron. Soc. Pacif. 113, 409-435 (2001).

3. Tremblay, P.-E. et al. Core crystallization and pile-up in the cooling sequence of evolving white dwarfs. Nature 565, 202-205 (2019).

4. Campbell, E. M. \& Hogan, W. J. The national ignition facility-applications for inertial fusion energy and high-energy-density science. Plasma Phys. Control. Fusion 41, B39 (1999).

5. Batani, D. et al. Development of the PETawatt Aquitaine Laser system and new perspectives in physics. Phys. Scr. T161, 014016 (2014).

6. Miquel, J.-L. \& Prene, M. LMJ \& PETAL status and program overview. Nucl. Fusion 59, 032005 (2019).

7. Rose, S. J. New experimental possibilities for measuring radiative opacity under conditions in the sun's interior. Plasma Phys. Control. Fusion 47, B735-B741 (2005).

8. Matzen, M. K. et al. The refurbished $\mathrm{Z}$ facility: capabilities and recent experiments. Acta Phys. Pol. A 115, 956 (2009).

9. Doeppner, T. et al. Demonstration of high performance in layered deuterium-tritium capsule implosions in uranium hohlraums at the National Ignition Facility. Phys. Rev. Lett. 115, 055001 (2015).

10. Regan, S. P. et al. Demonstration of fuel hot-spot pressure in excess of 50 Gbar for direct-drive, layered deuterium-tritium implosions on OMEGA. Phys. Rev. Lett. 117, 025001 (2016).

11. Le Pape, S. et al. Fusion energy output greater than the kinetic energy of an imploding shell at the National Ignition Facility. Phys. Rev. Lett. 120, 245003 (2018).

12. Doeppner, T. et al. Absolute equation-of-state measurement for polystyrene from 25 to 60 Mbar using a spherically converging shock wave. Phys. Rev. Lett. 121, 025001 (2018).

13. Gopalaswamy, V. et al. Tripled yield in direct-drive laser fusion through statistical modelling. Nature 565, 581-586 (2019).

14. Amendt, P. et al. Indirect-drive noncryogenic double-shell ignition targets for the National Ignition Facility: design and analysis. Phys. Plasmas 9, 2221 (2002).

15. Ping, Y. et al. Enhanced energy coupling for indirectly driven inertial confinement fusion. Nat. Phys. 15, 138-141 (2018).

16. Ciricosta, O. et al. Direct measurements of the ionization potential depression in a dense plasma. Phys. Rev. Lett. 109, 065002 (2012).

17. Hoarty, D. J. et al. Observations of the effect of ionization-potential depression in hot dense plasma. Phys. Rev. Lett. 110, 265003 (2013).

18. $\mathrm{Hu}, \mathrm{S}$. X. Continuum lowering and Fermi-surface rising in strongly coupled and degenerate plasmas. Phys. Rev. Lett. 119, 065001 (2017).

19. Hansen, S. B. et al. Fluorescence and absorption spectroscopy for warm dense matter studies and ICF plasma diagnostics. Phys. Plasmas 25, 056301 (2018).

20. Nilson, P. M. et al. Time-resolved measurements of hot-electron equilibration dynamics in high-intensity laser interactions with thin-foil solid targets. Phys. Rev. Lett. 108, 085002 (2012).

21. Smid, M. et al. Characterization of suprathermal electrons inside a laser accelerated plasma via highly-resolved Ka-emission. Nat. Commun. 10, 4212 (2019).

22. Rose, S. J. Calculations of the radiative opacity of laser-produced plasmas. J. Phys. B 25, 1667 (1992).

23. Iglesias, C. A. \& Rogers, F. J. Updated OPAL opacities. Astrophys. J. 464, 943 (1996).

24. Rose, S. J. The radiative opacity at the Sun centre-a code comparison study. J. Quant. Spectro. Rad. Trans. 71, 635-638 (2001).

25. Blancard, C., Cosse, P. \& Faussurier, G. Solar mixture opacity calculations using detailed configuration and level accounting treatments. Astrophys. J. 745, 10 (2012).

26. Colgan, J. et al. A new generation of Los alamos opacity tables. Astrophys. J. 817, 116 (2016).

27. Cederbaum, L. S., Zobeley, J. \& Tarantelli, F. Giant intermolecular decay and fragmentation of clusters. Phys. Rev. Lett. 79, 4778 (1997).

28. Schwartz, G. P., Fatehi, S., Saykally, R. J. \& Prendergast, D. Importance of electronic relaxation for inter-coulombic decay in aqueous systems. Phys. Rev. Lett. 105, 198102 (2010).

29. Rudenko, A. et al. Femtosecond response of polyatomic molecules to ultraintense hard X-rays. Nature 546, 129 (2017).

30. Lapp, M. Collision enforced transitions in cesium. Phys. Lett. 23, 553-554 (1966).

31. Gallagher, Alan \& Holstein, T. Collision-induced absorption in atomic electronic transitions. Phys. Rev. A 16, 2413-2431 (1977)

32. Ueda, K., Komatsu, T. \& Sato, Y. Observation of collision-induced-dipole absorption bands in strontium-rare gas mixtures. I. The $5 \mathrm{~s}-4 \mathrm{~d}$ bands. J. Chem. Phys. 91, 4495-4498 (1989).
33. Gonze, X. et al. Recent developments in the ABINIT software package. Comput. Phys. Commun. 205, 106-131 (2016)

34. Torrent, M., Jollet, F., Bottin, F., Zérah, G. \& Gonze, X. Implementation of the projector augmented-wave method in the ABINIT code: application to the study of iron under pressure. Comp. Mater. Sci. 42, 337-351 (2008).

35. Lambert, F., Clérouin, J. \& Zérah, G. Very-high-temperature molecular dynamics. Phys. Rev. E 73, 016403 (2006).

36. Recoules, V., Lambert, F., Decoster, A., Canaud, B. \& Clérouin, J. Ab Initio determination of thermal conductivity of dense hydrogen plasmas. Phys. Rev. Lett. 102, 075002 (2009).

37. Rose, S. Two-center electronic wave functions in plasmas. J. de. Phys. Colloq. 44, 159 (1983).

38. MacFarlane, J. J. et al. Simulation of the ionization dynamics of aluminum irradiated by intense short-pulse lasers. in (eds. Hammel, B., Meyerhofer, D., Meyer-ter-Vehn, J. and Azechi, H.) Proceedings of Inertial Fusion and Science Applications 2003. 457-460. (American Nuclear Society, LaGrange Park, IL, 2004).

39. Averbukh, V. \& Cederbaum, L. S. Interatomic electronic decay in endohedral fullerenes. Phys. Rev. Lett. 96, 053401 (2006).

40. Averbukh, V., Saalmann, U. \& Rost, J. M. Suppression of exponential electronic decay in a charged environment. Phys. Rev. Lett. 104, 233002 (2010).

41. Cooper, B. \& Averbukh, V. Single-photon laser-enabled auger spectroscopy for measuring attosecond electron-hole dynamics. Phys. Rev. Lett. 111, 083004 (2013).

42. Montgomery, D. S. et al. Design considerations for indirectly driven double shell capsules. Phys. Plasmas 25, 092706 (2018).

43. $\mathrm{Hu}, \mathrm{S}$. X. et al. Direct-drive double-shell implosion: a platform for burningplasma physics studies. Phys. Rev. E 100, 063204 (2019).

44. Perdew, J. P., Burke, K. \& Ernzerhof, M. Generalized gradient approximation made simple. Phys. Rev. Lett. 77, 3865-3868 (1996).

45. Pickard, C. J. \& Needs, R. J. Aluminium at terapascal pressures. Nat. Mater. 9 , 624 (2010).

46. Vinko, S. M., Ciricosta, O. \& Wark, J. S. Density functional theory calculation of continuum lowering in strongly coupled plasmas. Nat. Commun. 5, 3533 (2014).

47. Davis, P. et al. X-ray scattering measurements of dissociation-induced metallization of dynamically compressed deuterium. Nat. Commun. 7, 11189 (2016).

48. Baldereschi, A. Mean-value point in the Brillouin zone. Phys. Rev. B 7, 5212-5215 (1973).

49. Kubo, R. Statistical-mechanical theory of irreversible processes. I. General theory and simple applications to magnetic and conduction problems. J. Phys. Soc. Jpn. 12, 570-586 (1957).

50. Greenwood, D. A. The Boltzmann equation in the theory of electrical conduction in metals. Proc. Phys. Soc. Lond. 71, 585-596 (1958)

\section{Acknowledgements}

This material is based upon work supported by the US Department of Energy National Nuclear Security Administration under Award Number DE-NA0003856, the University of Rochester, and the New York State Energy Research and Development Authority. This work is partially supported by US National Science Foundation PHY Grant No. 1802964 for S.X.H. and V.V.K. This report was prepared as an account of work (for S.X.H., V.V. K., and P.M.N.) sponsored by an agency of the U.S. Government. Neither the U.S. Government nor any agency thereof, nor any of their employees, makes any warranty, express or implied, or assumes any legal liability or responsibility for the accuracy, completeness, or usefulness of any information, apparatus, product, or process disclosed, or represents that its use would not infringe privately owned rights. Reference herein to any specific commercial product, process, or service by trade name, trademark, manufacturer, or otherwise does not necessarily constitute or imply its endorsement, recommendation, or favoring by the U.S. Government or any agency thereof. The views and opinions of authors expressed herein do not necessarily state or reflect those of the U.S Government or any agency thereof.

\section{Author contributions}

S.X.H. conceived the project, performed the DFT calculations, and wrote the initial paper. V.V.K. created the all-electron PAW pseudopotentials and modified the ABINIT absorption code to calculate emission spectra. V.R., N.B., and M.T. have implemented the spin-orbital calculation for optical properties in ABINIT, and provided the code and help on running it. P.M.N. and S.X.H. discussed the possible experimental designs. All authors discussed the results and revised the paper.

\section{Competing interests}

The authors declare no competing interests. 


\section{Additional information}

Supplementary information is available for this paper at https://doi.org/10.1038/s41467020-15916-3.

Correspondence and requests for materials should be addressed to S.X.H.

Peer review information Nature Communications thanks Vitali Averbukh and the other, anonymous, reviewer(s) for their contribution to the peer review of this work. Peer reviewer reports are available.

Reprints and permission information is available at http://www.nature.com/reprints

Publisher's note Springer Nature remains neutral with regard to jurisdictional claims in published maps and institutional affiliations. (c) (i) Open Access This article is licensed under a Creative Commons Attribution 4.0 International License, which permits use, sharing, adaptation, distribution and reproduction in any medium or format, as long as you give appropriate credit to the original author(s) and the source, provide a link to the Creative Commons license, and indicate if changes were made. The images or other third party material in this article are included in the article's Creative Commons license, unless indicated otherwise in a credit line to the material. If material is not included in the article's Creative Commons license and your intended use is not permitted by statutory regulation or exceeds the permitted use, you will need to obtain permission directly from the copyright holder. To view a copy of this license, visit http://creativecommons.org/ licenses/by/4.0/.

(C) The Author(s) 2020 